\title{
Intelligence and robotics
}

\author{
Simon X. Yang \\ Advanced Robotics \& Intelligent Systems Laboratory, School of Engineering, University of Guelph, Guelph ON N1G 2W1, Canada.
}

Correspondence to: Prof. Simon X. Yang, Advanced Robotics \& Intelligent Systems Laboratory, School of Engineering, University of Guelph, 50 Stone Road East, Guelph ON N1G 2W1, Canada. E-mail: syang@uoguelph.ca

How to cite this article: Yang SX. Intelligence and robotics. Intell Robot 2021;1(1):1-2. https://dx.doi.org/10.20517/ir.2021.12

Received: 28 Sep 2021 Accepted: 28 Sep 2021 Available online: 9 Oct 2021

Academic Editor: Hao Zhang Copy Editor: Xi-Jun Chen Production Editor: Xi-Jun Chen

Welcome to the inaugural issue of Intelligence \& Robotics. It is my great pleasure and honor to be involved as the Editor-in-Chief and founder of the new journal Intelligence \& Robotics. We are delighted with and highly grateful to the many colleagues and friends with diversified expertise in intelligence and robotics worldwide to support the Journal and serve on our editorial board, including advisory board members, associate editors, and youth editorial board members. We are also immensely grateful for the tremendous support from our publisher OAE Publishing Inc., USA.

Biological inspiration provides the basis for many aspects of robotics. Robot manipulators were first developed to approximate the reaching and grasping abilities of the human arm. Walking machines were attempts to perform some of the locomotion and gait features of living things. Studies of intelligence have made significant progress in understanding the biological intelligence of various species and developing innovative artificial and bionic strategies, mechanisms, algorithms, and technologies, with diversified applications to various fields, particularly robotics. On the other hand, robotics studies have made remarkable progress in theoretical investment and real-world applications to various industries. There is a general movement toward service-oriented intelligent robotic systems that require the ability to adapt to complex dynamic situations and handle various uncertainties, such as self-driving cars. Intelligence will be essential to these robotic systems performing successfully, as living organisms can adapt to changing environments that are only partially known and not predictable. Therefore, it is essential to bring experts from the fields of intelligence and robotics together to accomplish original and innovative achievements. 
Intelligence \& Robotics publishes top-quality unpublished original technical and non-technical applicationfocused articles on the general areas of intelligence and robotics, particularly the interdisciplinary area of intelligence and robotics. The Journal seeks to publish articles dealing with the theory, design, analysis, evaluation, and applications of intelligence and robotics, ranging from system modeling, data analysis, algorithms, and software/hardware development of various intelligent and/or robotic systems.

The scope of the Journal includes, but is not limited to, (1) the biological, bio-inspired, and artificial intelligence, such as neural networks, fuzzy systems, evolutionary algorithms, genetic algorithms, ant colony optimization, particle swarm optimization, artificial immune systems, simulated annealing, smart gambler strategy, expert systems, and various other intelligent methodologies; (2) the design, modeling, analysis, evaluation, and implementation of various robotic systems, such as mobile, aerial, surface, and underwater robotic systems; (3) the real-time information acquisition, multi-sensor fusion, data analysis, localization and map building, path planning, tracking, and control for various robotic systems; and (4) the cooperation, coordination, communication, teleoperation, and human-machine interactions of multiple robotic systems. In addition, the Journal would be interested in distributed development and maintenance of real-world intelligent and robotic systems by multidisciplinary teams of scientists and engineers.

This journal aims to provide a platform for all experts, professionals, and scholars with creative contributions to get together and share some inspiring ideas and accomplish outstanding achievements in the general fields of intelligence and robotics.

\section{DECLARATIONS}

\section{Authors' contributions}

The author contributed solely to the article.

\section{Availability of data and materials}

Not applicable.

\section{Financial support and sponsorship}

None.

\section{Conflicts of interest}

The author declared that there are no conflicts of interest.

\section{Ethical approval and consent to participate}

Not applicable.

\section{Consent for publication}

Not applicable.

\section{Copyright}

() The Author(s) 2021. 